\title{
Health-Related Quality of Life and Health Resource Utilization in Patients with Primary Immunodeficiency Disease Prior to and Following 12 Months of Immunoglobulin G Treatment
}

\author{
John Routes ${ }^{1,2}$ - Beatriz Tavares Costa-Carvalho ${ }^{3}$ - Bodo Grimbacher ${ }^{4,5} \cdot$ Kenneth Paris $^{6}$. \\ Hans D. Ochs ${ }^{7}$ - Alexandra Filipovich ${ }^{8}$ - Mary Hintermeyer ${ }^{1} \cdot$ Karina Mescouto de Melo $^{3,5}$.

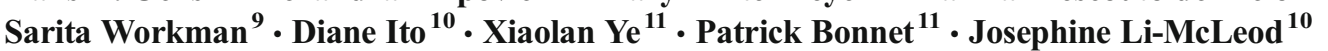

Received: 6 October 2015 / Accepted: 29 March 2016/Published online: 18 April 2016

(C) The Author(s) 2016. This article is published with open access at Springerlink.com

\begin{abstract}
Purpose Health-related quality of life (HRQOL) has not been examined in patients with predominant antibody deficiency both pre- and post-immunoglobulin $\mathrm{G}(\mathrm{IgG})$ treatment initiation. HRQOL and health resource utilization (HRU) were assessed in newly diagnosed patients with primary immunodeficiency disease (PIDD) pre- and 12 months post-IgG treatment initiation. Methods Adults (age $\geq 18$ years) completed the 36-item Short Form Health Survey, version 2; pediatric patients (PP)/caregivers
\end{abstract}

Xiaolan Ye and Patrick Bonnet are former employees of Baxalta US, Inc.

John Routes

jroutes@mcw.edu

1 Medical College of Wisconsin and the Children's Research Institute, Milwaukee, WI, USA

2 Section of Allergy and Clinical Immunology, Department of Pediatrics, Medical College of Wisconsin, 9000 W. Wisconsin Ave., Milwaukee, WI 53226-4874, USA

3 Universidade Federal de Sao Paulo, Sao Paulo, Brazil

4 Institute of Immunity \& Transplantation, Royal Free Hospital, University College London, London, UK

5 Center for Chronic Immunodeficiency, University Hospital Freiburg, Freiburg im Breisgau, Germany

6 LSU Health Sciences Center, Children's Hospital New Orleans, New Orleans, LA, USA

7 Seattle Children's Research Institute and University of Washington, Seattle, WA, USA

8 Cincinnati Children's, Cincinnati, OH, USA

9 The Royal Free London NHS Foundation Trust, London, UK

10 Baxalta US, Inc, Cambridge, MA, USA

11 Baxalta US, Inc, Bannockburn, IL, USA completed the Pediatric Quality of Life Inventory (PedsQL). Scores were compared with normative data from the US general population (GP) and patients with other chronic conditions (OCC).

Results Seventeen adult patients (APs), 8 PPs, and 8 caregivers completed baseline assessments. APs had significantly lower baseline mean physical component summary scores versus GP (37.4 vs 50.5, $p<0.01)$ adults with chronic back pain $(44.1, p<0.05)$ or cancer $(44.4, p<0.05)$ and lower mental component summary scores versus GP (41.6 vs 49.2 , $p<0.05$ ). PPs had lower PedsQL total (63.1 vs 82.7), physical summary (64.5 vs 84.5$)$, and psychosocial summary (62.5 vs 81.7) scores versus GP. Post-IgG treatment, 14 APs, 6 PPs, and 8 caregivers completed assessments. Hospital admissions ( 0.2 versus $1.8, p<0.01)$, serious infections ( 3.3 versus 10.9 , $p<0.01$ ) and antibiotic prescriptions (3.0 versus $7.1 ; p<0.01$ ) decreased significantly overall. While APs reported significant improvement in role-physical $(p=0.01)$, general health $(p<0.01)$, and social functioning $(p=0.02)$ and caregivers in vitality $(p<0.01)$, PPs did not.

Conclusions Pre-IgG treatment, patients with PIDD experienced diminished HRQOL versus GP and patients with OCC; post-treatment, HRU decreased and certain HRQOL aspects improved for APs and caregivers.

Keywords Primary immunodeficiency · antibody deficiency . health-related quality of life $\cdot$ health resource utilization, short form health survey (SF-36) · pediatric quality of life inventory (PedsQL)

\section{Introduction}

Primary immunodeficiency diseases (PIDDs), a heterogeneous group of disorders that include antibody deficiencies 
[1-4], are characterized by increased susceptibility to infections [1]. Patients with predominant antibody deficiencies can present in infancy or adulthood [5] and are particularly vulnerable to bacterial infections that may result in chronic lung disease, bronchiectasis, and gastrointestinal symptoms $[2,4$, 6-8]. Affected patients experience a range of symptoms that require lifelong treatment. Antibody replacement with immunoglobulin $G(\operatorname{Ig} G)$ is the most commonly utilized prophylactic/therapeutic approach for the management of antibody deficiency disorders, and IgG administered intravenously or subcutaneously has been shown to safely and effectively reduce the frequency and severity of infections in these patients [9-16].

PIDD imposes a significant disease burden on patients, including limitations in work, play, or normal physical activity $[17,18]$. Quality of life, including health-related quality of life (HRQOL), in patients with PIDD can be significantly affected by delays in diagnosis [1, 19-24]. Currently, these delays are considerable, as data from a patient survey in the United States indicated that the average time from the onset of symptoms associated with PIDD to the time of diagnosis is 12.4 years [17], similar to or longer than published reports of average delays of 12.5 years [25], 4.7 years [26], and 4.4 years [27] from other countries. Furthermore, following treatment initiation, patients have also reported lower HRQOL compared with the general population [18, 23, 28-32]. However, details of the HRQOL burden patients with PIDD experience both before diagnosis and after IgG treatment initiation are lacking.

In this study we investigated the burden of disease among patients and caregivers of pediatric patients newly diagnosed with PIDD, focusing on patients with impaired antibody production, and assessed the impact of IgG treatment on patient quality of life. Our primary goal was to gain insight into the effect of infections and impairments on HRQOL prior to and after initiation of treatment with $\mathrm{IgG}$ in patients with predominantly antibody deficiencies. We present comparisons of HRQOL between patients with PIDD and age- and sexmatched patients with other chronic conditions and the general US population. In addition, we present an assessment of healthcare utilization in the 6-month period preceding the start of and 12-months following treatment initiation with IgG.

\section{Methods}

\section{Study Design}

A 12-month, prospective, uncontrolled, open-label, observational study was initiated in 2010 to assess the burden of disease among patients with newly diagnosed predominant antibody deficiencies and, following initiation with $\operatorname{IgG}$ treatment, the impact of treatment on quality of life and health resource utilization. Patients were recruited from six Jeffrey
Modell Foundation treatment centers in the United States, Brazil, and the United Kingdom. Participating study sites offered a financial incentive to study participants in the form of (1) two $\$ 25$ checks, (2) up to $\$ 50$ for reimbursement of transportation costs, or (3) no financial incentive, depending on the local regulatory requirements. The appropriate institutional review board or ethics committee at each site or country approved the study protocol.

\section{Participants}

The study focused on patients with predominant antibody deficiencies in the absence of significant T-cell defects. Eligible patients were recently diagnosed with or suspected of having common variable immunodeficiency (CVID), XLA or autosomal recessive agammaglobulinemia, or specific antibody deficiency. Diagnoses of PIDD that require treatment beyond regular IgG replacement (eg, severe combined immunodeficiency and Wiskott-Aldrich Syndrome) were excluded. All diagnoses of PIDD were confirmed by laboratory testing and all patients were symptomatic (ie, had recurrent infections) and deemed suitable for IgG treatment. Both pediatric (age $<18$ years) and adult (age $\geq 18$ years) patients who qualified for IgG replacement but had not yet started therapy were included. Patients with a life expectancy of $<1$ year, dementia or mental incapacity, or with comorbidities or other conditions that would interfere with the data interpretation were excluded. Patients who were currently receiving $\operatorname{IgG}$ or had received IgG in the recent past were also not eligible for the study. Patients or their caregivers provided written informed consent and were enrolled in the study from September 2010 to December 2012. IgG treatment regimen and dosing intervals were per the discretion of the treating physician.

\section{Data Collection}

Patients were assessed at two time points. Baseline assessment occurred after confirmation of the diagnosis and during the 2 weeks before first IgG infusion or on the first day of treatment. The follow-up assessment took place 12 months after initiation of $\operatorname{IgG}$ treatment ( \pm 1 month), with the last patient follow-up completed in December 2013. At each assessment, serious infections, or other acute or chronic infections (eg, acute chronic sinusitis; bronchitis; bacterial pneumonia; bacteremia or sepsis; osteomyelitis or septic arthritis; visceral abscess; bacterial meningitis; and ear infection) were captured, and patients completed self-administered questionnaires to evaluate quality of life and health resource utilization. Pediatric patients aged $8-17$ years completed the questionnaire with the help of their caregiver (parent/guardian). Caregivers completed the questionnaire on behalf of patients aged $<8$ years. Collected data were entered into an electronic database for analysis. 
Adult patients and caregivers of pediatric patients completed the 36-item Short Form Health Survey, version 2 (SF-36v2) $[33,34]$. The SF-36v2 survey is a validated and frequently used $[35,36]$ measure of HRQOL that evaluates eight domains related to functional health status and emotional well-being. These eight domains can be categorized into higher-order clusters of physical health (physical function, role-physical, bodily pain, general health) and mental health (vitality, social function, role - emotional, and mental health).

Pediatric patients (age $<18$ years) were evaluated with the Pediatric Quality of Life Inventory (PedsQL) measurement instrument [37]. The PedsQL survey is a validated, reliable [38] measure of HRQOL specifically for the pediatric population that evaluates physical, emotional, social, and schoolrelated parameters.

A health resource utilization questionnaire was also administered at baseline to collect information on hospitalizations, emergency room visits, antibiotic use and days missed from work or school in the 6 months prior to enrollment. Patients were also given a diary for recording healthcare utilization during the 12-month study period.

\section{Statistical Analysis}

The primary endpoint for the analysis was the baseline and the 12-month follow up quality-of-life (QOL) score. Secondary endpoints included occurrence of hospitalization, emergency room visits, office visits for PIDD-related events, and use of medications, such as antibiotics for these time points. Descriptive statistical analyses were carried out for all primary and secondary endpoint parameters.

Using the normative data available for the SF-36v2, comparative samples were age- and sex-matched using separate least squares multiple regression models for each QOL scale and summary measure. While the SF$36 \mathrm{v} 2$ normative database is based on US respondents only, research has shown that the US-based norms are valid and appropriate for interpreting scores of respondents from other countries [39].

The SF-36v2 normative database allowed for comparisons to a general US population as well as subgroups within that population with the following chronic conditions: rheumatoid arthritis, chronic obstructive pulmonary disease (COPD), congestive heart failure (CHF), cancer, and chronic back pain. A univariate analysis of variance model was used to test differences between the SF-36v2 scores for patients with PIDD and patients from the comparative groups. The PedsQL has published normative data from healthy and chronic conditions, including asthma, cancer, diabetes, and rheumatology for comparison purposes [37]. Because the normative data for the PedsQL are in published form only, $95 \%$ confidence intervals were used to assess differences between scores for patients with PIDD and patients from the comparative groups.

A power calculation to determine the appropriate sample size for this study was not conducted due to the lack of historical results to estimate the potential effect size for the HRQOL endpoints and due to the exploratory nature of the study without a formal hypothesis formulated or tested. The study aimed to include 30 or more patients with PIDD; due to the rarity of PIDD and the challenge of finding newly diagnosed patients naïve to IG therapy, such a number was considered to be reasonably attainable, while adding valuable information to conduct an exploratory analysis of the stated endpoints.

\section{Determination of Clinically Relevant Differences}

Changes in SF-36 scores prior to and following IgG treatment initiation were compared with known minimally important differences (MID) for each category [40]. Changes that met or exceeded the MID threshold were considered to be clinically meaningful.

\section{Results}

\section{Patient Characteristics}

A total of 31 patients (11 each from the United States and Brazil, and 9 from the United Kingdom) were enrolled in the study. The enrolled population included 21 (66.7\%) adult patients and $10(33.3 \%)$ pediatric patients. Six patients discontinued early due to treatment noncompliance (1 adult), home relocation (1 pediatric), and unknown reasons (3 adults and 1 pediatric). Baseline assessments were completed by 17 adult patients, 8 pediatric patients, and 8 caregivers, while 12-month assessments were completed by 14 adults, 6 pediatric patients, and 8 caregivers. Missing data from 2 of the pediatric patients precluded their inclusion in the follow-up analyses.

Baseline characteristics for the 25 patients and 8 caregivers included in the analysis are summarized in Table 1 . The mean age was 47.7 years (standard deviation $[\mathrm{SD}]=12.9$ ) for adult patients and 5.1 years $(\mathrm{SD}=4.8)$ for pediatric patients. CVID was the most frequently occurring diagnosis amongst the adults (14/21, 66.6\%), while hypogammaglobulinemia was the most common diagnosis among the pediatric patients $(4 / 8,50 \%)$.

\section{QOL Assessment in Adult Patients}

Seventeen of the 21 adults enrolled completed SF-36v2 assessments prior to IVIG treatment initiation (baseline); the 3 adult patients who did not complete the 
Table 1 Patient characteristics

\begin{tabular}{|c|c|c|c|}
\hline \multirow[t]{2}{*}{ Characteristic } & \multicolumn{2}{|l|}{ Patients } & \multirow{2}{*}{$\begin{array}{l}\text { Caregivers } \\
\text { (Mothers) } \\
(N=8)\end{array}$} \\
\hline & $\begin{array}{l}\text { Adult } \\
\text { (age } \geq 18 \text { years) } \\
(N=17)\end{array}$ & $\begin{array}{l}\text { Pediatric } \\
(\text { age }<18 \text { years }) \\
(N=8)\end{array}$ & \\
\hline \multicolumn{4}{|l|}{ Age, years } \\
\hline Mean (SD) & $47.1(13.2)$ & $5.5(5.4)$ & $32.8(8.6)$ \\
\hline Min-max & $24-67$ & $0-17$ & $23-47$ \\
\hline \multicolumn{4}{|l|}{ Sex, n (\%) } \\
\hline Male & $6(35.3)$ & $5(62.5)$ & $0(0)$ \\
\hline Female & $11(64.7)$ & $3(37.5)$ & $8(100)$ \\
\hline \multicolumn{4}{|l|}{ Diagnosis, n (\%) } \\
\hline CVID & $11(64.7)$ & $2(25)$ & $\mathrm{N} / \mathrm{A}$ \\
\hline Other $^{\mathrm{a}}$ & $6(35.3)$ & $6(75)$ & N/A \\
\hline Agammaglobulinemia & $1(5.9)$ & $0(0)$ & N/A \\
\hline Hypogammaglobulinemia & $2(11.7)$ & $4(50)$ & N/A \\
\hline Specific antibody deficiency & $3(17.6)$ & $2(25)$ & N/A \\
\hline \multicolumn{4}{|l|}{ Working status, n (\%) } \\
\hline Work full-time ( $\geq 35 \mathrm{~h} /$ week) & $9(52.9)$ & N/A & $2(25.0)$ \\
\hline Work part-time $(<35 \mathrm{~h} /$ week $)$ & $2(11.8)$ & N/A & $2(12.5)$ \\
\hline Stay-at-home parent & $0(0)$ & N/A & $1(12.5)$ \\
\hline Disabled & $1(5.9)$ & N/A & $1(12.5)$ \\
\hline Unemployed & $0(0)$ & N/A & $3(37.5)$ \\
\hline Retired & $4(23.5)$ & N/A & $0(0)$ \\
\hline Other & $1(5.9)$ & N/A & $0(0)$ \\
\hline \multicolumn{4}{|l|}{ Marital status, n (\%) } \\
\hline Single & $4(23.5)$ & N/A & $1(12.5)$ \\
\hline Married & $103(58.8)$ & N/A & $4(50.0)$ \\
\hline Divorced & $1(5.9)$ & N/A & $3(37.5)$ \\
\hline Other & $2(11.8)$ & N/A & $0(0)$ \\
\hline \multicolumn{4}{|l|}{ Race } \\
\hline White or Caucasion & $17(100.0)$ & $7(87.5)$ & N/A \\
\hline African-American & $0(0)$ & $1(12.5)$ & N/A \\
\hline \multicolumn{4}{|l|}{ Location of infusions } \\
\hline Home & $2(11.8)$ & $0(0)$ & N/A \\
\hline Infusion center & $15(88.2)$ & $8(100)$ & N/A \\
\hline
\end{tabular}

${ }^{a}$ Patients in this category were all deemed suitable candidates for the current study and for IgG replacement treatment CVID common variable immunodeficiency (CVID), IgG immunoglobulin G), max maximum), min minimum), $N / A$ not applicable), $S D$ standard deviation

assessments after 12 months of treatment were excluded from the baseline SF-36v2 analyses. Prior to treatment initiation, adult patients who were diagnosed with PIDD had diminished HRQOL compared with the general US population and patients with other chronic conditions (Fig. 1a and b). The mean Physical Component Summary (PCS) score for adult patients $(N=17)$ with PIDD was 37.4, which was significantly lower than in the general US population $(50.5 ; N=4,024 ; p<0.01)$, and in patients with various types of cancer, excluding skin cancer $(44.4, N=311 ; p<0.05)$ or chronic back pain $(44.1, N=893 ; p<0.05)$ (see Fig. 1a). In the physical health domains of the SF-36v2 (physical functioning, role-physical, bodily pain, general health), adult patients with PIDD prior to treatment had significantly lower scores for all 4 domains compared with the general US population $(p<0.05)$, and compared with patients suffering from other chronic conditions for rolephysical ( $p<0.05$ for rheumatoid arthritis, cancer, chronic back pain) and general health $(p<0.05$ for 
Fig. 1 SF-36 physical (a) and mental (b) summary and domain scores $^{\mathrm{a}}$ for adult patients with PIDD compared with patients with other chronic conditions and US general population. ${ }^{\mathrm{a}}$ Physical functioning, role physical, bodily pain, and general health are domains of the physical component scores; vitality, social functioning, role emotional, and mental health are domains of the mental component score. ${ }^{*} p<0.05$ compared with PIDD sample. ${ }^{*} p<0.01$ compared with PIDD sample. CHF, congestive heart failure; $\mathrm{COPD}$, chronic obstructive pulmonary disease; IgG, immunoglobulin G; MID, minimally important difference; PIDD, primary immunodeficiency diseases; SF36 , short form health survey; US, United States
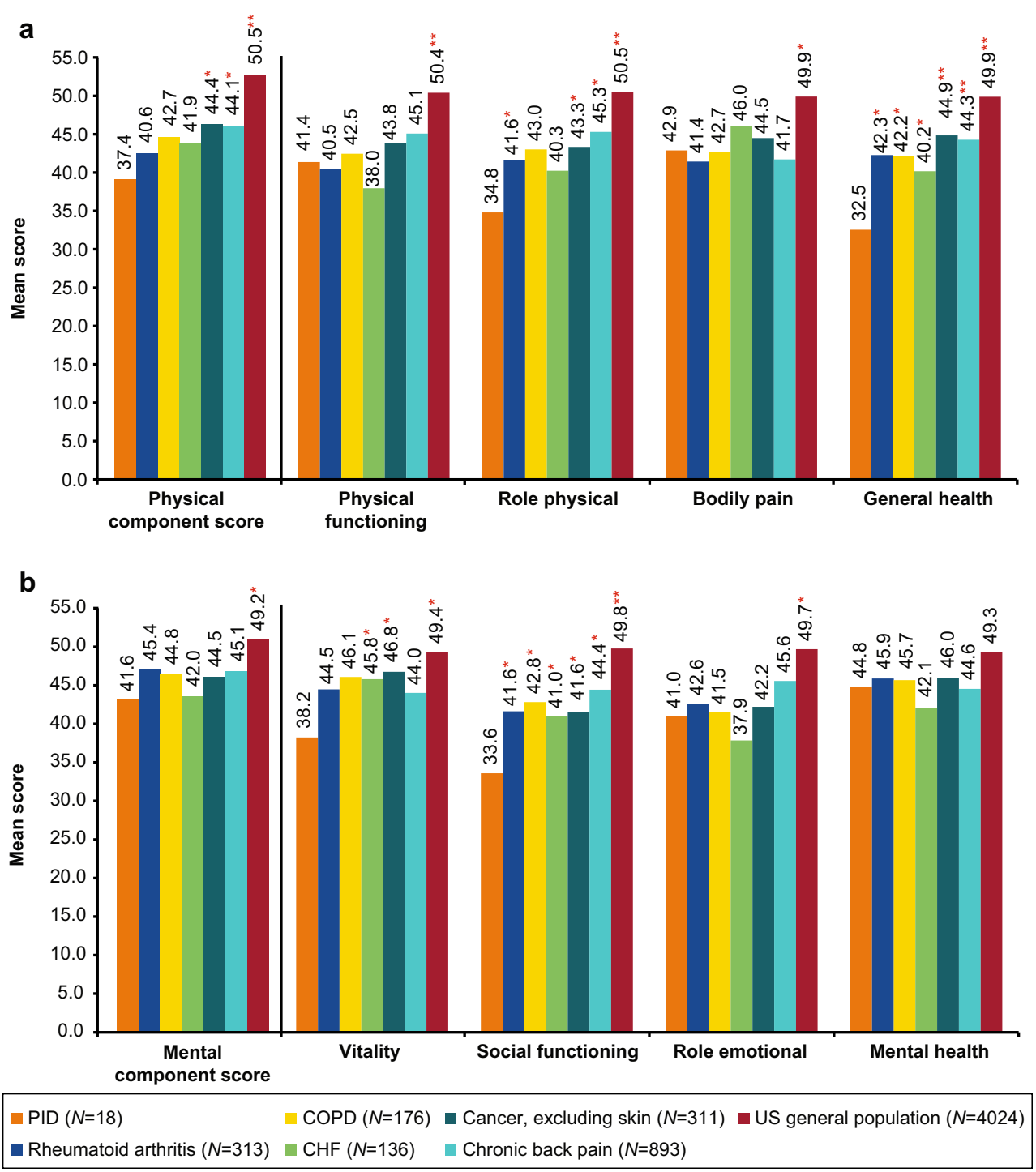

rheumatoid arthritis, COPD, and CHF; $p<0.01$ for cancer and chronic back pain).

Similarly, the mean Mental Component Summary (MCS) score was significantly lower for adult patients with PIDD $(41.6 ; n=17)$ compared with the general US population (49.2, $N=4,024 ; p<0.05$ ) (see Fig. 1b). Furthermore, the MCS for adult patients with PIDD was lower, but not statistically significant, than MCS values reported by patients with other chronic conditions (including rheumatoid arthritis, COPD, CHF, cancer, and chronic back pain). For the mental health domains of the SF-36v2, adult patients with PIDD experienced significantly diminished $(p<0.05)$ vitality $(38.2$ vs. 49.4), social functioning (33.6 vs. 49.8), and role-emotional (41.0 vs. 49.7), but not mental health, compared with the general US population. Adult patients with PIDD also reported significantly lower scores for social functioning $(p<0.05$ for rheumatoid arthritis,
COPD, CHF, cancer, and chronic back pain) and vitality $(p<0.05$ for $\mathrm{CHF}$ and cancer) relative to age-matched patients with chronic diseases.

Of the 17 adult patients assessed for QOL at baseline, 14 were assessed twelve months after IgG treatment initiation. Comparing baseline and follow up data for these 14 patients, increases in both PCS (from 36.9 to 43.2; Fig. 2a) and MCS (from 46.0 to 49.0; Fig. 2b) were observed. While these differences were not significant, the change in PCS (6.3) after IgG treatment exceeded the MID of 3.8 and may be clinically relevant. Furthermore, significant and clinically relevant increases were observed in a number of physical and mental individual domain scores, including rolephysical $(p=0.01$; observed change $=10.6, M I D=4.0)$, general health $(p<0.01 ;$ observed change $=9.1$, $M I D=7.0)$, and social functioning $(p=0.02$; observed change $=8.8, M I D=6.2)$ (see Figs. $2 \mathrm{a}$ and $\mathrm{b}$ ). 
Fig. 2 SF-36 physical (a) and mental (b) component and domain scores ${ }^{\mathrm{a}}$ of adult patients with PIDD at baseline and at 12 months following $\operatorname{IgG}$ treatment initiation. ${ }^{\text {a }}$ Physical functioning, role physical, bodily pain, and general health are domains of the physical component scores; vitality, social functioning, role emotional, and mental health are domains of the mental component score.

SF-36 US general population norms, $\Delta$, difference. MID, minimally important difference; ns, not significant; PIDD, primary

immunodeficiency disease; SF36, 36-item Short Form Health Survey; US, United States

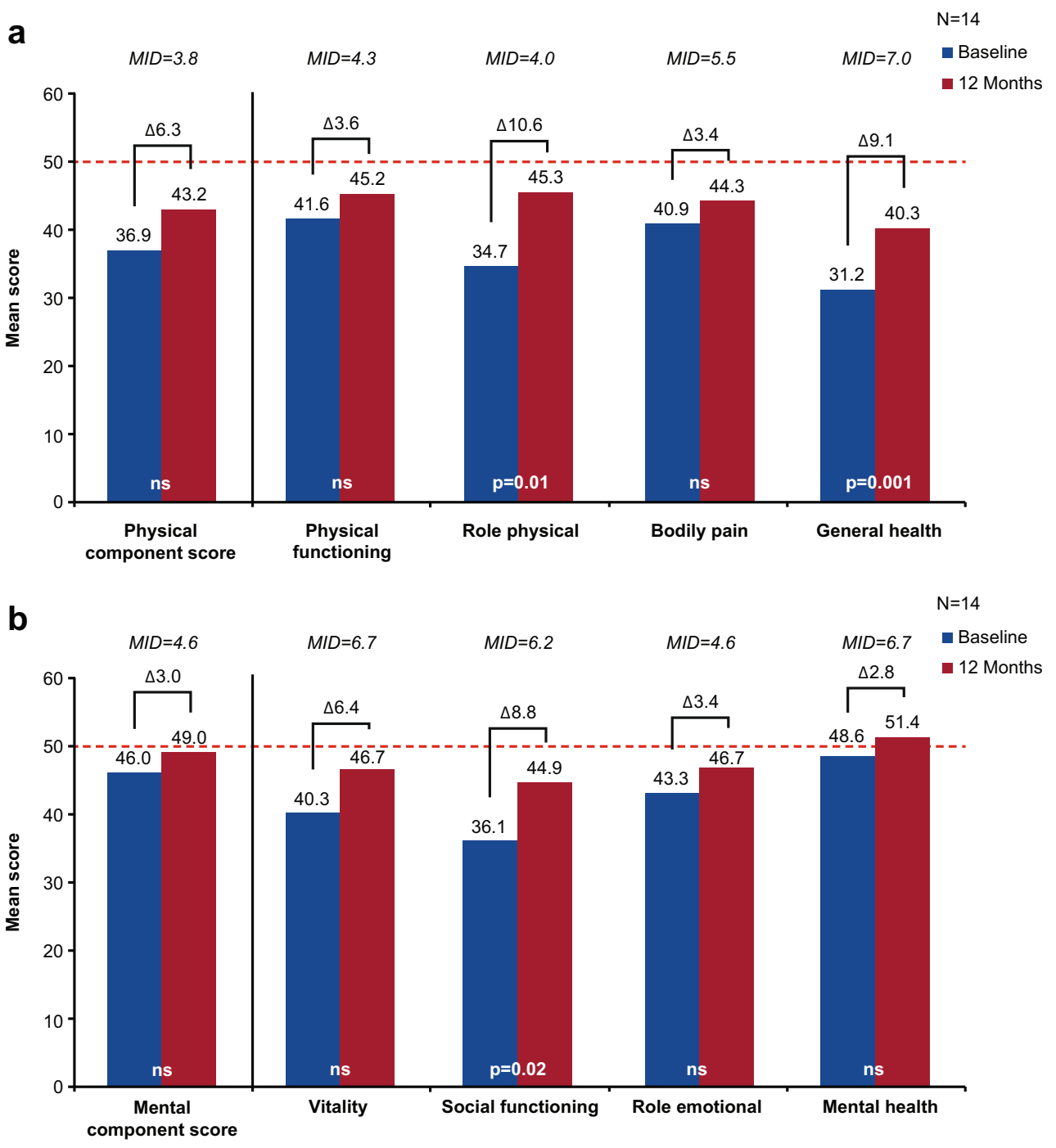

\section{QOL Assessment in Pediatric Patients}

Eight of the 10 enrolled pediatric patients completed PedsQL assessments at baseline; the 2 patients who did not complete both assessments were excluded from the PedsQL analyses. According to baseline PedsQL scores, pediatric patients diagnosed with PIDD experienced diminished HRQOL compared with the healthy pediatric population (Fig. 3). Those with PIDD $(N=8)$ had significantly lower $(p=0.05)$ PedsQL total scores (63.1 [95 \% confidence interval; CI: 48.4-77.9] vs. 82.7 [95 \% CI: 82.4-83.0]), physical summary scores (64.5 [95 \% CI: 46.7-82.2] vs. 84.5 [95 \% CI: 84.1-84.9]), and psychosocial summary scores (62.5 [95 \% CI: 48.0-77.0] vs. 81.7 [95\% CI: 81.3-82.0]) compared with the general US population $(N=9,430)$. The total scores, physical summary scores, and psychosocial summary scores were also lower for pediatric patients with PIDD relative to those with asthma $(N=157)$, diabetes $(N=307)$, cancer $(N=561)$, and rheumatology conditions $(N=357)$, with the exception of physical summary score comparability of PIDD with rheumatology conditions.

Of the 8 pediatric patients with PIDD assessed at baseline, 6 completed assessments 12 months following IgG treatment initiation. No significant or clinically meaningful differences in total, physical summary, or psychosocial summary scores were reported (Fig. 4).

Figure 5 illustrates the change in PedsQL scores from baseline to 12 months for each individual pediatric patient. Examination of these individual changes shows a wide variation in the PedsQL scores between patients.

\section{Health Resource Utilization in Adult and Pediatric Patients}

Health resource utilization among patients with PIDD prior to and after IgG treatment initiation is summarized in Table 2. Adult and pediatric patients as a group reported a significantly lower mean number of hospital admissions $(n=25 ; 1.8$ vs 
Fig. 3 PedsQL total, physical, and psychosocial summary scores of pediatric patients with PIDD compared with pediatric patients with other chronic conditions and the US healthy population. The black bars represent the $95 \%$ confidence intervals. PedsQL, pediatric quality of life; PIDD, primary immunodeficiency disease; US, United States

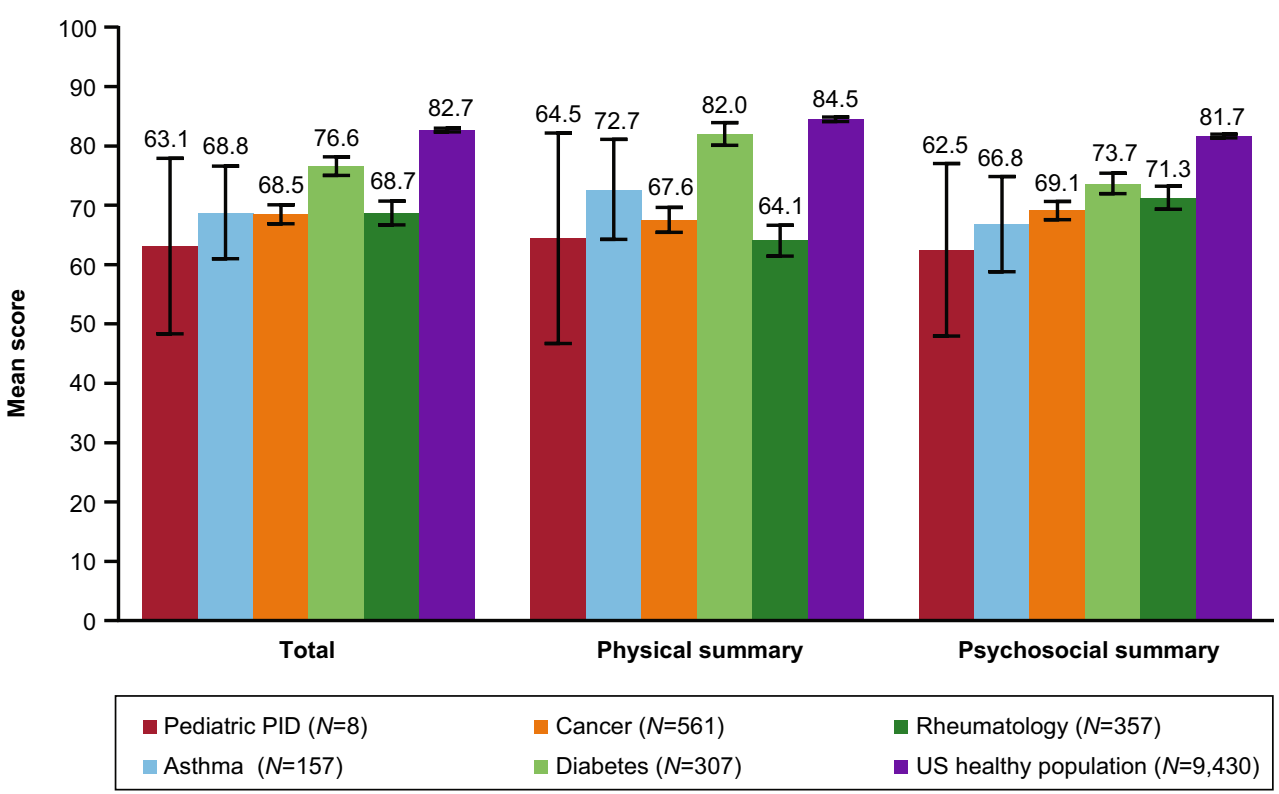

$0.21 ; p<0.01)$, serious infections $(n=18 ; 10.9$ vs 3.3 ; $p<0.01)$, and antibiotic prescriptions $(n=22 ; 7.1$ vs 3.0 ; $p<0.01)$ after initiation of $\mathrm{IgG}$ treatment compared with baseline. An adult-only sub analysis further showed a significant decrease in mean emergency room visits associated with $\mathrm{IgG}$ treatment $(n=17 ; 1.8$ at baseline vs 0.4 post-treatment initiation, $p=0.04$ ). Additionally, pediatric patients had significantly fewer mean number of unscheduled office visits $(n=7 ; 20.0$ vs $8.3, p=0.01)$ after treatment initiation compared with baseline.

\section{Patient Productivity}

Adult and pediatric patients with PIDD experienced a reduction in productivity in the 6 months preceding treatment initiation. After initiation of $\operatorname{IgG}$ treatment, adult patients $(n=12)$ reported a non-significant increase in days missed from work/school (mean 25.5 days prior to IgG treatment and 37.4 days after 12 months of $\operatorname{IgG}$ treatment), while pediatric patients $(n=4)$ reported 63.5 and 46.0 days missed from school, prior to and after IgG treatment initiation, respectively (see Table 2).

\section{QOL Assessment in Caregivers of Pediatric Patients}

Eight caregivers completed SF-36v2 assessments at baseline and 12 months following IgG treatment initiation; the 2 caregivers who did not complete both assessments were excluded from the SF-36 analyses. While caregivers did not report a significant difference in PCS (Fig. 6a) or MCS (Fig. 6b) following IgG treatment, MCS was numerically higher $(38.0 \mathrm{vs}$ 30.9 ) and clinically relevant (observed change $=7.1$; $M I D=4.6$ ) after 12 months of treatment compared with baseline. Furthermore, compared with baseline, caregivers also

Fig. 4 PedsQL scores among pediatric patients with PIDD at baseline and at 12 months following $\mathrm{IgG}$ treatment initiation. ${ }^{\mathrm{a}} \mathrm{MIDs}$ were not available for summary and component scores. IgG immunoglobulin G; MID, minimally important difference; ns, not significant; PedsQL, Pediatric Quality of Life; PIDD, primary immunodeficiency diseases; US, United States

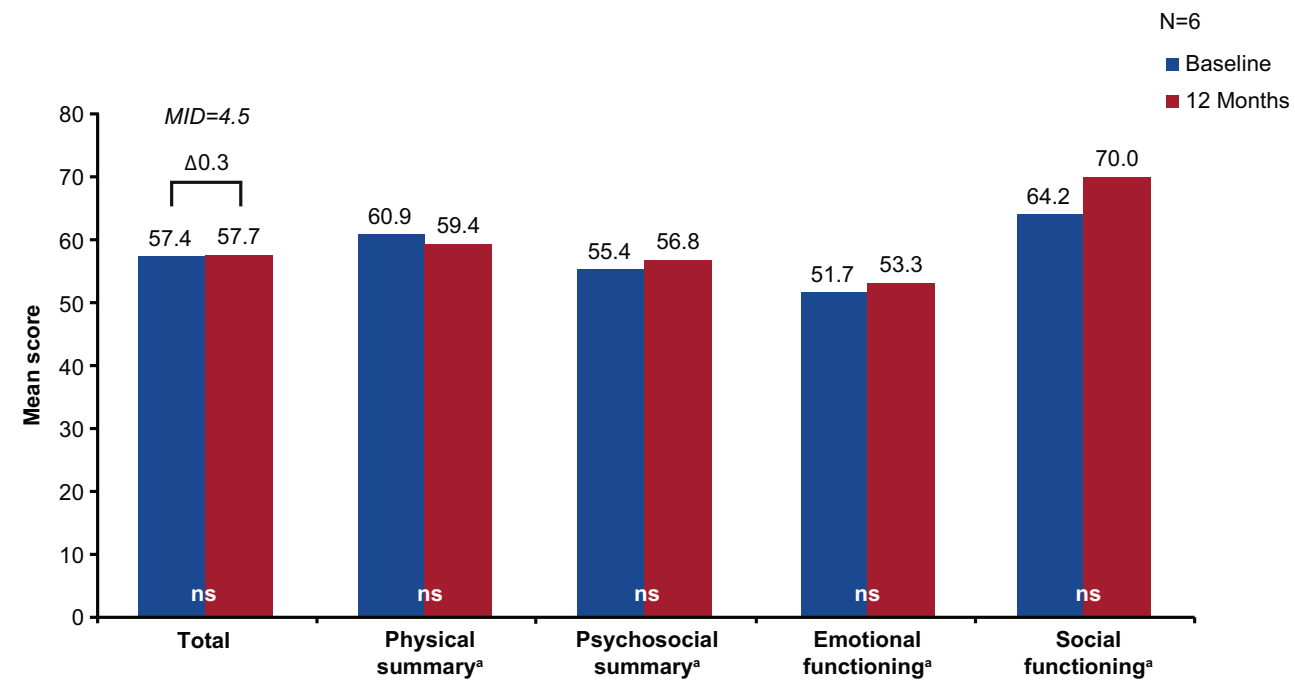


Fig. 5 PedsQL scores among individual pediatric patients with PIDD at baseline and at 12 months following IgG treatment initiation. $\mathrm{IgG}$, immunoglobulin G; PedsQL, Pediatric Quality of Life; PIDD, primary immunodeficiency diseases
- Patient 1 (age $\leq 5$ years; United States)

- Patient 2 (age 6-12 years; United States)

- Patient 3 (age 13-17 years; United States)

- Patient 4 (age 6-12 years; International)

- Patient 5 (age $\leq 5$ years; International)

- Patient 6 (age 6-12 years; International)

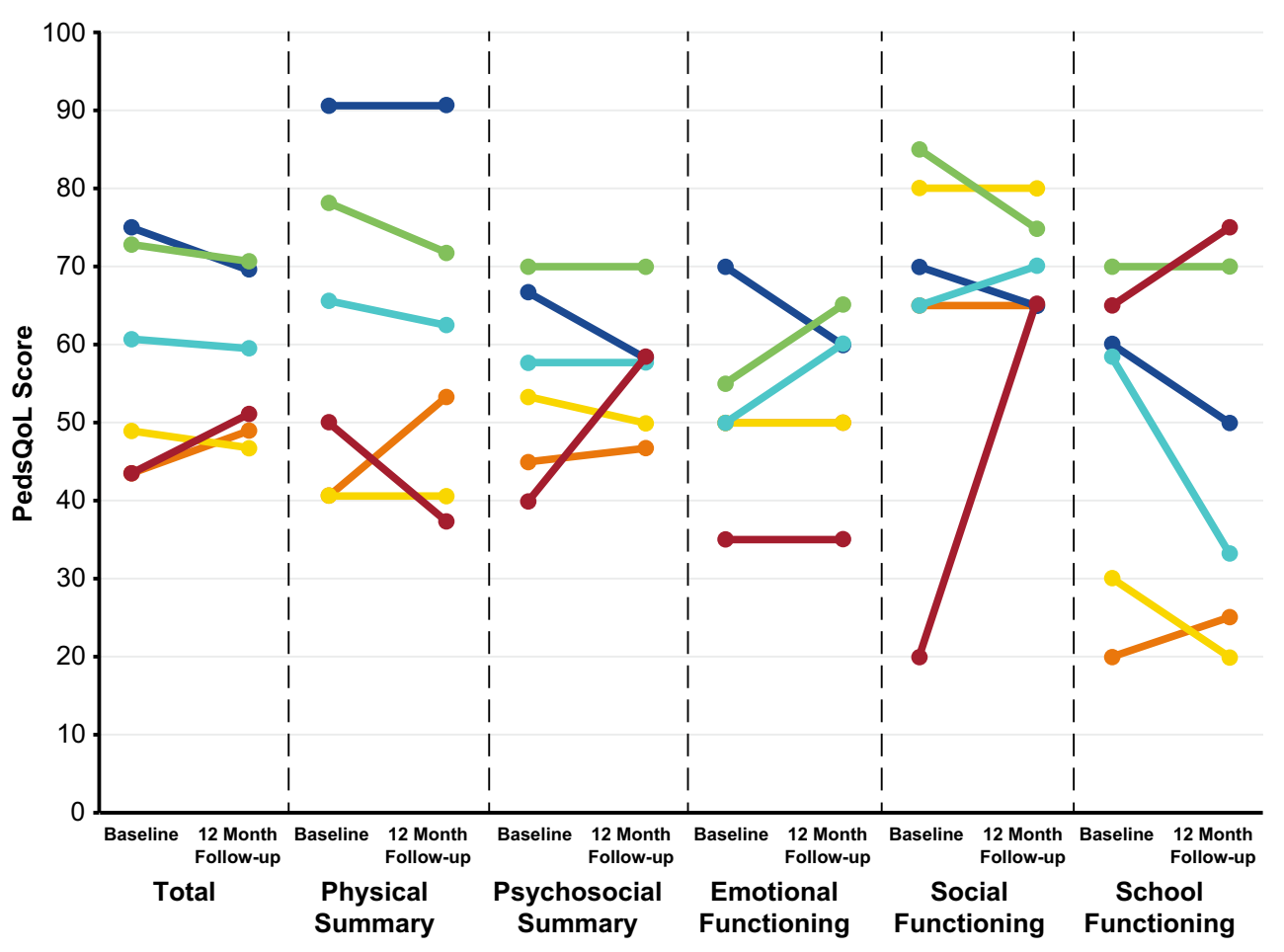

reported a significantly higher $(47.0$ vs. $38.8, p<0.01)$ and clinically relevant (observed change $=8.2, M I D=6.7$ ) domain score for vitality after treatment (see Fig. 6b). Clinically relevant (but not statistically significant) increases in social functioning (observed change $=6.9, M I D=6.2$ ) and mental health (observed change $=7.8, M I D=6.7$ ) were also reported (see Fig. 6b).

\section{Discussion}

Recent data suggest that although the prevalence of PIDD is higher than once thought [41], patients continue to experience significant delays in diagnosis $[17,25,26]$. The present study sought to gain insight into the burden of disease among newly diagnosed patients with antibody deficiencies and to explore HRQOL and HRU following the initiation of $\mathrm{IgG}$ treatment. The SF-36 and PedsQL surveys used to assess HRQOL in adult and pediatric patients, respectively, in the current survey have previously been used in other studies of patients with PIDD [18, 21, 22, 28-30, 34]. The results presented herein suggest that adult and pediatric patients with PIDD have diminished HRQOL and productivity prior to the initiation of
IgG treatment, and that following 12 months of IgG, adult (but not pediatric) patients experienced improved HRQOL, as reflected by significant and clinically relevant increases in 2 physical health and 1 mental health domain scores in the SF36 survey. Furthermore, the data also suggest improved HRQOL for pediatric patients' caregivers, who reported a significant increase in their own vitality following 12 months of IgG treatment of their children. Notably, clinically meaningful increases in social functioning and mental health domain scores were also observed for caregivers. In addition, significant decreases were reported in a number of HRU categories for both adult and pediatric patients. Compared with baseline, however, the number of missed days at work (adults) or school (pediatric patients) was not significantly different following IgG treatment.

The wide variation in the PedsQL scores observed between individual pediatric patients (see Fig. 5) suggests that while some pediatric patients reported an improvement after 12 months of treatment, others reported no change or worsening of their HRQOL. However, these data are difficult to interpret due to the low number of pediatric patients in our study population. Although the wide variation may reflect a true variation within the pediatric PIDD population, the disparity 
Table 2 Mean health resource utilization per patient before and 12 months after IgG treatment initiation

\begin{tabular}{|c|c|c|c|c|c|c|c|c|}
\hline \multirow[t]{2}{*}{ Health Resource Utilization } & \multicolumn{4}{|c|}{ Adult Patients (Age $\geq 18$ Years) } & \multicolumn{4}{|c|}{ Pediatric Patients (Age $<18$ Years) } \\
\hline & $\mathrm{n}$ & $\begin{array}{l}\text { Baseline (12-Months } \\
\text { Pre-treatment), } \\
\text { Mean (SD) }\end{array}$ & $\begin{array}{l}\text { 12- Months, } \\
\text { Mean (SD) }\end{array}$ & p-value & $\mathrm{n}$ & $\begin{array}{l}\text { Baseline (12-Months } \\
\text { Pre-treatment), } \\
\text { Mean (SD) }\end{array}$ & 12 Months, Mean (SD) & p-value \\
\hline Regularly scheduled office visits & 16 & $7.1(7.8)$ & $8.9(8.7)$ & NS & 7 & $10.6(5.3)$ & $11.3(5.7)$ & NS \\
\hline Unscheduled office visits & 17 & $11.6(15.6)$ & $3.8(5.4)$ & NS & 7 & $20.0(9.2)$ & $8.3(6.8)$ & 0.01 \\
\hline Emergency room visits & 17 & $1.8(2.4)$ & $0.4(0.7)$ & $<0.05$ & 7 & $6.3(8.3)$ & $1.9(3.3)$ & NS \\
\hline Hospital admissions & 17 & $1.4(2.4)$ & $0.2(0.4)$ & 0.05 & 8 & $2.8(2.1)$ & $0.4(0.5)$ & 0.01 \\
\hline Length of stay ${ }^{\mathrm{b}}$ & - & - & - & & 3 & $46.0(46.1)$ & $7.7(8.1)$ & NS \\
\hline Number of serious infections ${ }^{c}$ & 12 & $8.0(7.2)$ & $2.5(1.6)$ & $<0.05$ & 6 & $16.7(9.0)$ & $5.0(2.1)$ & $<0.05$ \\
\hline Days missed from work or school ${ }^{\mathrm{d}}$ & 12 & $25.5(36.9)$ & $37.4(103.4)$ & NS & 4 & $63.5(77.8)$ & $46.0(36.5)$ & NS \\
\hline Number of antibiotic prescriptions & 16 & $5.5(4.0)$ & $2.5(2.3)$ & $<0.01$ & 6 & $11.3(9.0)$ & $4.2(2.6)$ & NS \\
\hline
\end{tabular}

${ }^{a}$ Pre-enrollment data was annualized

${ }^{\mathrm{b}}$ Only 1 adult patient had length-of-stay data

${ }^{\mathrm{c}}$ Serious infections defined as: acute or chronic sinusitis, bronchitis, pneumonia, ear infection, or other acute or chronic infection

${ }^{\mathrm{d}}$ Four adult patients who were retired were excluded from this analysis

$E R$ emergency room, $\operatorname{Ig} G$ immunoglobulin G, $N S$ not significant, $S D$ standard deviation

Fig. 6 SF-36 physical (a) and mental (b) component and domain scores $^{\mathrm{a}}$ for caregivers of pediatric patients with PIDD at baseline and at 12 months following $\mathrm{IgG}$ treatment initiation. "Physical functioning, role physical, bodily pain, and general health are domains of the physical component scores; vitality, social functioning, role emotional, and mental health are domains of the mental component score. - SF-36 US general population norms. $\Delta$, difference. MID, minimally important difference; ns, not significant; PIDD, primary immunodeficiency disease; SF36, 36-item Short Form Health Survey; US, United States

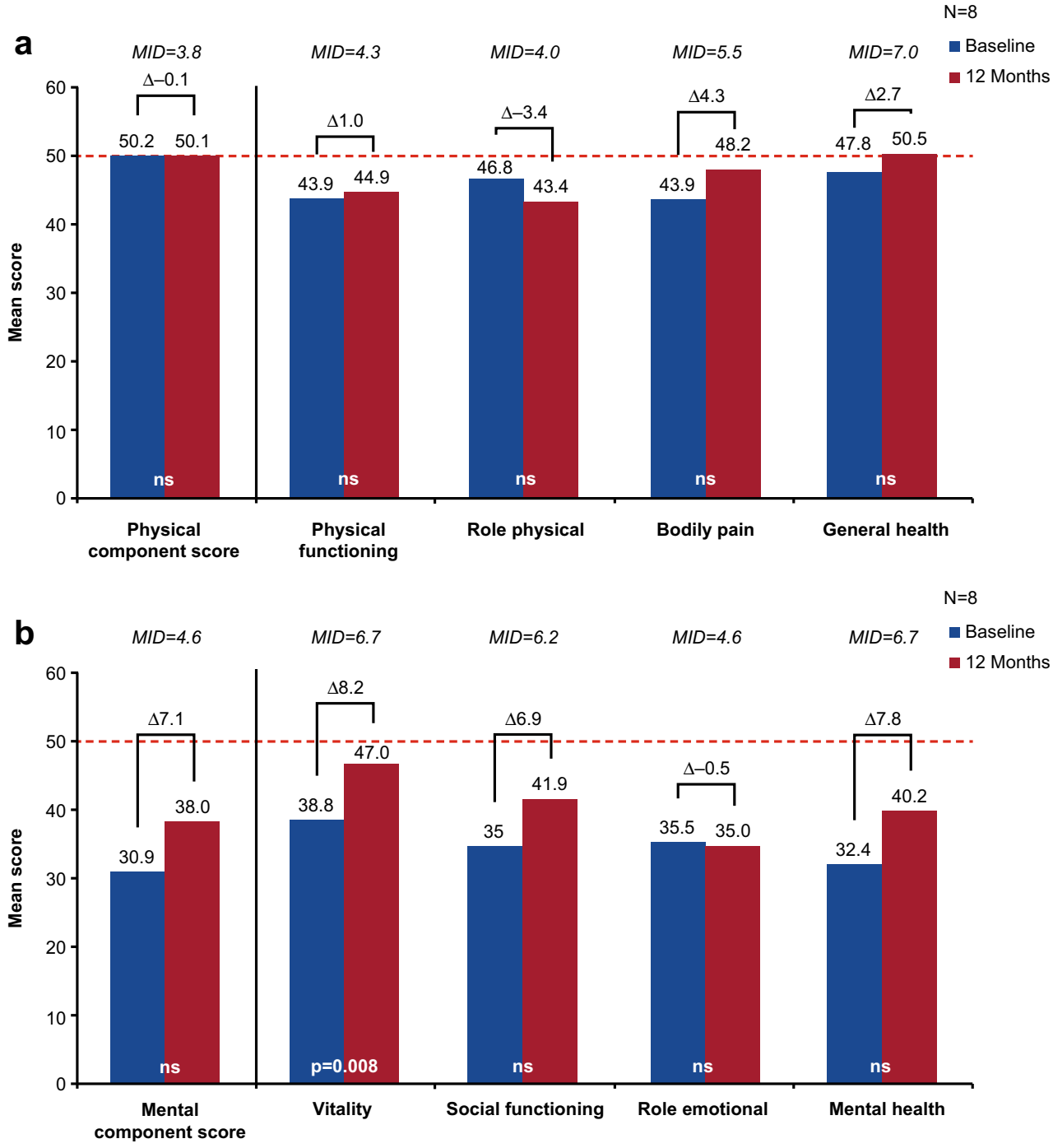


may also reflect an aberration within our small study sample; specific trends may be elucidated with a larger sample size.

While several studies have investigated HRQOL in adult and pediatric patients with PIDD following IgG replacement [18, 21-23, 28-32], none have compared HRQOL in the same cohort prior to and after treatment initiation. In general, data from previous studies have shown a lower HRQOL in patients with PIDD compared with the general population, including adult patients with CVID [18] and select IgA deficiency [28]. Similar results were reported from studies focusing on pediatric patients in which significantly lower PedsQL scores were observed in those with PIDD compared with healthy individuals [29, 31, 32]. However, in a survey of 25 adult males with XLA, numerically, but not significantly, lower QOL scores, compared with the general US population, were reported [23].

In the current study, QOL and HRU were assessed within the same patient cohort both prior to PIDD diagnosis and following 12 months of IgG replacement therapy. At baseline, adults with PIDD had significantly lower PCS and MCS (SF36) compared with the general US population and significantly lower PCS compared with patients with chronic back pain and cancer. The mental burden, on the other hand, was similar between adults with PIDD and patients with other chronic conditions. Similarly, pediatric patients diagnosed with PIDD experienced a substantial HRQOL burden of disease, as was evident from the lower PedsQL scores than the general US population. When compared with pediatric patients with other chronic diseases, including cancer, asthma, and rheumatologic diseases, scores were either similar or lower.

Patients were followed after 12 months of IgG treatment. After treatment, patients documented lower HRU with regards to hospital admissions, serious infections, and antibiotic prescriptions. Adult patients reported a significant increase in the role-physical and general health domain scores of PCS and in the vitality domain of the MCS. These results are consistent with other studies that have noted an inverse relationship between persistence of symptoms (ie, serious infections, chronic diarrhea) and SF-36 scores [18, 30]. While previous studies have primarily indicated a QOL deficit in patients with PIDD who are already receiving treatment compared with healthy populations or patients with other chronic diseases, the current study indicates that adult patients do experience an improvement in QOL following IgG treatment initiation. However, this study did not collect interim assessment data (ie, within the 12 months of treatment); therefore, an opportunity was missed to compare differences in improvement during, eg, the first 6 months of $\mathrm{IgG}$ treatment to the last 6 months of IgG treatment.

In addition to evaluating patients with PIDD, the current study also surveyed caregivers (mothers) of pediatric patients with PIDD. Notably, caregivers reported a significant increase in the vitality domain as well as clinically relevant differences in the social functioning and mental health domains of MCS after their children initiated IgG replacement, suggesting that the QOL benefit may extend beyond the patients themselves. However, both pediatric and caregiver results should be interpreted with caution due to the particularly low number of subjects in these analyses (as further illustrated by the variation in individual pediatric PedsQL data in Fig. 5).

The present study focused on patients with antibody deficiencies, and the small sample size (particularly the low number of pediatric patients and their caregivers) presented here may not accurately reflect the general population of patients with PIDD. Further, since a power calculation was not conducted, this study may have been underpowered to adequately detect the true effect of treatment on patient HRQOL. Because of the small number of patients in the current study, additional analyses are limited. For example, it is not statistically plausible to compare the socioeconomic status and geographical areas of the patients. It is also important to consider that selfreported survey results are always subject to potential bias because patients and caregivers may not accurately remember or report details of their condition. In the current analysis, we compared scores from patients with PIDD (prior to and after treatment with $\mathrm{IgG}$ ) to the SF-36v2 normative database without information regarding the treatment status of patients with chronic conditions included in the database. If some or all of the patients with chronic conditions had received treatments, it might impact their HRQOL and subsequently influence our comparison. Furthermore, while prior research has shown that using the US-based SF-36v2 norms for interpreting scores across adult respondents from different countries is appropriate (between-score correlations of 0.98-1.00 for physical and mental components in all 9 European countries evaluated) [39], the comparability with the patients from our sample from other countries may be limited because the SF-36v2 normative database contains only US respondents. Similarly, the PedsQL survey has been translated (with cultural adaptations) and validated for use in numerous countries (PedsQL: http:// pedsql.org/pedsq12.html). Although the PedsQL has been validated among different ethnic and racial backgrounds within a US population [38], to our knowledge no crosscountry validation studies of the PedsQL survey have been published.

\section{Conclusions}

In the current analysis, prior to IgG replacement, adult and pediatric patients with PIDD suffered from diminished HRQOL compared with the general US population and patients with other chronic diseases. In addition, patients experienced diminished productivity due to the number of work and/or school days that were missed because of their condition. Twelve months after IgG treatment initiation, adult 
patients and caregivers of pediatric patients reported an improvement in QOL. Additionally, HRU decreased for the overall population, including pediatric patients.

Acknowledgments This manuscript is dedicated to the memory of Dr. Richard Schiff, Baxata US, Inc, physician, expert immunologist and colleague, whose work and contributions to developing more effective treatments for patients with immunodeficiencies is well recognized and highly appreciated.

Writing and editorial assistance were provided by BlueMomentum, an Ashfield Company, part of UDG Healthcare plc, and funded by Baxalta US, Inc Healthcare.

\section{Compliance with Ethical Standards}

Conflicts of Interest Beatriz Tavares Costa-Carvalho: none. Alexandra Filipovich: none.

Bodo Grimbacher: Head of Jeffrey Modell Center (funded by Baxalta US, Inc).

Mary Hintermeyer: none.

Diane Ito: employee, Baxalta US, Inc.

Karina Mescouto de Melo: none.

Hans D. Ochs: member of advisory boards, CSL-Behring and Baxalta US, Inc.

Kenneth Paris: Clinical trial investigator, advisory board participant, speaker on diagnosis of primary immunodeficiency: Baxalta US, Inc.

John Routes: none.

Sarita Workman: Baxalta US, Inc, BPL, Biotest, Grifols, CSL - Behring, Octapharma, Viropharma, and Shire have all provided educational grants for attendance at various educational meetings over the last 5 years, not exceeding $£ 10,000$ - only paying for transportation, registration and accommodation.

Xiaolan Ye: former employee, Baxalta US, Inc.

Patrick Bonnet: former employee, Baxalta US, Inc.

Josephine Li-McLeod: employee, Baxalta US, Inc.

Open Access This article is distributed under the terms of the Creative Commons Attribution 4.0 International License (http:// creativecommons.org/licenses/by/4.0/), which permits unrestricted use, distribution, and reproduction in any medium, provided you give appropriate credit to the original author(s) and the source, provide a link to the Creative Commons license, and indicate if changes were made.

\section{References}

1. Picard C, Al-Herz W, Bousfiha A, Casanova JL, Chatila T, Conley ME, et al. Primary immunodeficiency diseases: an update on the classification from the international union of immunological societies expert committee for primary immunodeficiency 2015. J Clin Immunol. 2015;35(8):696-726.

2. Wood P, Stanworth S, Burton J, Jones A, Peckham DG, Green T, et al. Recognition, clinical diagnosis and management of patients with primary antibody deficiencies: a systematic review. Clin Exp Immunol. 2007;149:410-23.

3. Parvaneh N, Casanova JL, Notarangelo LD, Conley ME. Primary immunodeficiencies: a rapidly evolving story. J Allergy Clin Immunol. 2013;131:314-23.

4. Cooper MA, Pommering TL, Korányi K. Primary immunodeficiencies. Am Fam Physician. 2003;68:2001-8.
5. Ballow M. Primary immunodeficiency disorders: antibody deficiency. J Allergy Clin Immunol. 2002;109:581-91.

6. Chen Y, Stirling RG, Paul E, Hore-Lacy F, Thompson BR, Douglass JA. Longitudinal decline in lung function in patients with primary immunoglobulin deficiencies. J Allergy Clin Immunol. 2011;127:1414-7.

7. Rich AL, Le Jeune IR, McDermott L, Kinnear WJ. Serial lung function tests in primary immune deficiency. Clin Exp Immunol. 2008;151:110-3.

8. Boujaoude Z, Arya R, Rafferty W, Dammert P. Organising pneumonia in common variable immunodeficiency. BMJ Case Rep. 2013. doi:10.1136/bcr-2013-008905.

9. Skull S, Kemp A. Treatment of hypogammaglobulinaemia with intravenous immunoglobulin, 1973-93. Arch Dis Child. 1996;74: 527-30.

10. Ochs HD, Gupta S, Kiessling P, Nicolay U, Berger M. Safety and efficacy of self-administered subcutaneous immunoglobulin in patients with primary immunodeficiency diseases. J Clin Immunol. 2006;26:265-73.

11. Gardulf A, Nicolay U, Asensio O, Bernatowska E, Böck A, Carvalho BC, et al. Rapid subcutaneous IgG replacement therapy is effective and safe in children and adults with primary immunodeficiencies - a prospective, multi-national study. J Clin Immunol. 2006;26:177-85.

12. Chapel HM, Spickett GP, Ericson D, Engl W, Eibl M, Bjorkander J. The comparison of the efficacy and safety of intravenous versus subcutaneous immunoglobulin replacement therapy. J Clin Immunol. 2000;20:94-100.

13. Buckley RH, Schiff RI. The use of intravenous immune globulin in immunodeficiency disease. N Engl J Med. 1991;325:110-7.

14. Wasserman RL, Irani AM, Tracy J, Tsoukas C, Stark D, Levy R, et al. Pharmacokinetics and safety of subcutaneous immune globulin (human), $10 \%$ caprylate/chromatography purified in patients with primary immunodeficiency disease. Clin Exp Immunol. 2010;161:518-26.

15. Wasserman RL, Melamed I, Kobrynski L, Strausbaugh SD, Stein MR, Sharkhawy M, et al. Efficacy, safety, and pharmacokinetics of a $10 \%$ liquid immune globulin preparation (GAMMAGARD LIQUID, $10 \%$ ) administered subcutaneously in subjects with primary immunodeficiency disease. J Clin Immunol. 2011;31:323-31.

16. Wasserman RL, Melamed I, Stein MR, et al. Recombinant human hyaluronidase-facilitated subcutaneous infusion of human immunoglobulins for primary immunodeficiency. J Allergy Clin Immunol. 2012;130(4):951-7.

17. Immune Deficiency Foundation. Primary immune deficiency diseases in America: the third national survey of patients. 2007. http:// primaryimmune.org/idf-survey-research-center/idf-surveys/patientsurveys/. Accessed 29 May 2014.

18. Quinti I, Di Pietro C, Martini H, Pesce AM, Lombardi F, Baumghartner $\mathrm{M}$, et al. Health related quality of life in common variable immunodeficiency. Yonsei Med J. 2012;53:603-10.

19. Chan A, Scalchunes C, Boyle M, Puck JM. Early vs. delayed diagnosis of severe combined immunodeficiency: a family perspective survey. Clin Immunol. 2011;138:3-8.

20. Buckley RH. Immune deficiency foundation diagnostic and clinical care guidelines for primary immunodeficiency diseases, 2 nd ed. 2009. http://primaryimmune.org/wp-content/uploads/2011/04/ IDF-Diagnostic-Clinical-Care-Guidelines-for-PrimaryImmunodeficiency-Diseases-2nd-Edition.pdf. Accessed 29 May 2014.

21. Nicolay U, Kiessling P, Berger M, Gupta S, Yel L, Roifman CM, et al. Health-related quality of life and treatment satisfaction in North American patients with primary immunodeficiency diseases receiving subcutaneous $\mathrm{IgG}$ self-infusions at home. J Clin Immunol. 2006;26:65-72. 
22. Gardulf A, Borte M, Ochs HD, Nicolay U, Vivaglobin Clinical Study Group. Prognostic factors for health-related quality of life in adults and children with primary antibody deficiencies receiving SCIG home therapy. Clin Immunol. 2008; 126:81-8.

23. Winkelstein JA, Conley ME, James C, Howard V, Boyle J. Adults with X-linked agammaglobulinemia: impact of disease on daily lives, quality of life, educational and socioeconomic status, knowledge of inheritance, and reproductive attitudes. Medicine (Baltimore). 2008;87:253-8.

24. Daly PB, Evans JH, Kobayashi RH, Kobayashi AL, et al. Homebased immunoglobulin infusion therapy: quality of life and patient health perceptions. Ann Allerty. 1991;67(5):504-10.

25. Ramírez-Vargas N, Arablin-Oropeza SE, Mojica-Martínez D, Yamazaki-Nakashimada MA, de la Luz G-CM, Terán-Juárez LM, et al. Clinical and immunological features of common variable immunodeficiency in Mexican patients. Allergol Immunopathol (Madr). 2014;42:235-40.

26. Guaní-Guerra E, García-Ramírez UN, Jiménez-Romero AI, Velázquez-Ávalos JM, Gallardo-Martínez G, Mendoza-Espinoza FJ. Primary immunodeficiency diseases at reference and highspecialty hospitals in the state of Guanajuato, Mexico. Biomed Res Int. 2013;2013:187254.

27. Seymour B, Miles J, Haeney M. Primary antibody deficiency and diagnostic delay. J Clin Pathol. 2005;58:546-7.

28. Jörgensen GH, Gardulf A, Sigurdsson MI, Arnlaugsson S, et al. Health-related quality of life (HRQL) in immunodeficient adults with selective IgA deficiency compared with age- and gendermatched controls and identification of risk factors for poor HRQL. Qual Life Res. 2014;23(2):645-58.

29. Kuburovic NB, Pasic S, Susic G, Stevanovic D, et al. Health-related quality of life, anxiety, and depressive symptoms in children with primary immunodeficiencies. Patient Prefer Adherence. 2014;8: 323-30.

30. Aghamohammadi A, Montazeri A, Abolhassani H, Saroukhani S, et al. Health-related quality of life in primary antibody deficiency. Iran J Allergy Asthma Immunol. 2011;10(1):47-51.
31. Zebracki K, Palermo TM, Hostoffer R, Duff K, et al. Health-related quality of life of children with primary immunodeficiency disease: a comparison study. Ann Allergy Asthma Immunol. 2004;93(6):557-61.

32. Soresina A, Nacinovich R, Bomba M, et al. The quality of life of children and adolescents with X-linked agammaglobulinemia. J Clin Immunol. 2009;29:501-7.

33. Ware Jr JE, Kosinski M, Gandek B. SF-36 health survey: manual and interpretation guide. Lincoln, RI: QualityMetric; 2000.

34. Ware Jr JE, Sherbourne CD. The MOS 36-item short-form health survey (SF-36). I. Conceptual framework and item selection. Med Care. 1992;30:473-83.

35. McHorney CA, Ware Jr JE, Raczek AE. The MOS 36-item shortform health survey (SF-36): II. Psychometric and clinical tests of validity in measuring physical and mental health constructs. Med Care. 1993;31:247-63.

36. Scoggins JF, Patrick DL. The use of patient-reported outcomes instruments in registered clinical trials: evidence from ClinicalTrials.gov. Contemp Clin Trials. 2009;30:289-92.

37. Varni JW, Limbers CA, Burwinkle TM. Impaired health-related quality of life in children and adolescents with chronic conditions: a comparative analysis of 10 disease clusters and 33 disease categories/severities utilizing the PedsQL 4.0 Generic Core Scales. Health Qual Life Outcomes. 2007;5:43.

38. Varni JW, Burwinkle TM, Seid M. The PedsQL as a pediatric patient-reported outcome: reliability and validity of the PedsQL Measurement Model in 25,000 children. Expert Rev Pharmacoecon Outcomes Res. 2005;5:705-19.

39. Ware Jr JE, Gandek B, Kosinski M, Aaronson NK, Apolone G, Brazier J, et al. The equivalence of SF-36 summary health scores estimated using standard and country-specific algorithms in 10 countries: results from the IQOLA Project. International Quality of Life Assessment. J Clin Epidemiol. 1998;51:1167-70.

40. Maruish, ME (Ed.). User's manual for the SF-36v2 health survey 3rd ed. Lincoln, RI: Quality Metric Incorporated.

41. Kobrynski L, Powell RW, Bowen S. Prevalence and morbidity of primary immunodeficiency diseases, United States 2001-2007. J Clin Immunol. 2014;34(8):954-61. 